\title{
A Secure and Efficient Off-line Electronic Payment System for Wireless Networks
}

\author{
H. Oros, C. Popescu
}

\author{
Horea Oros, Constantin Popescu \\ Department of Mathematics and Computer Science, University of Oradea \\ Str. Universitatii 1, Oradea, Romania \\ E-mail: \{horos,cpopescu\}@uoradea.ro
}

\begin{abstract}
An electronic cash system allows the exchange of digital coins with value assured by the bank's signature and with concealed user identity. In an electronic cash system, a user can withdraw coins from the bank and then spends each coin anonymously and unlinkably. In this paper we propose a secure and efficient off-line electronic payment system based on bilinear pairings and group signature schemes. The anonymity of the customer is revocable by a trustee in case of a dispute. Because the amount of communication in the payment protocol is about 480 bits, the proposed off-line electronic payment system can be used in wireless networks with limited bandwidth.

Keywords: Electronic payment system, bilinear pairings, group signatures, membership certificate.
\end{abstract}

\section{Introduction}

Chaum suggested the first electronic cash system [5] in 1982. In this system the technique of blind signatures was used to guarantee the privacy of users. Various extended systems have been proposed, which provide functionalities such as anonymity, double spending prevention, unforgeability, untraceability and efficiency [1], [4], [8]. Off-line electronic cash systems were first introduced in [6] and then developed further in [9], [10], [11], [12]. In off-line systems the bank's involvement in the payment transaction between a customer and a merchant was eliminated. Customers withdraw electronic coins from the bank and use them to pay a merchant (a shop). The merchant subsequently deposits the coins back to the bank.

In this paper we propose a secure off-line electronic payment system based on bilinear pairings and group signature schemes. In order to construct our electronic cash system, we use the group signature scheme of D. Yao and R. Tamassia [16] and the blind signature of Schnorr [13]. Due to the low amount of communication in the payment protocol that is about 480 bits, our off-line electronic payment system can be used in wireless networks with limited bandwidth.

The rest of this paper is organized as follows. In the next section we present our off-line electronic cash system. Furthermore, we discuss some aspects of security and efficiency in section 3. Finally, section 4 concludes the work of this paper.

\section{The Proposed Off-Line Electronic Payment System}

An e-cash system is a set of parties with their interactions, exchanging money and goods. A typical e-cash system has three parties:

- Customer: purchases goods or services from the merchant using the e-cash.

- Merchant: sells goods or services to the customer, and deposits the e-cash to the bank.

- Bank: issues the e-cash and maintains the bank account for customers and merchants.

And there are also three protocols: withdrawal, payment and deposit. A customer withdraws electronic coins from the bank and pays the coins to a merchant. Finally, the merchant deposits the paid coins to the bank.

Our electronic payment system consists of four types of participants: customers, merchants, banks and trusted parties. The customers honestly withdraw money from the bank and pay money to the merchant. The merchants get money from customers and deposit it in the bank. The banks manage customer accounts, issue and redeem money. The bank can legally trace a dishonest customer with the help of the trusted parties. An e-cash system is 
anonymous if the bank in collaboration with the merchant cannot trace the coin to the customer. The system is off-line if during payment the merchant does not communicate with the bank.

In our off-line electronic cash system, all customers who open a bank account form a group and a trusted party is the group manager. When a customer wants to withdraw an electronic coin from his account, the bank applies a blind signature protocol [13] to this coin and decreases appropriate amount from the customer's account. Everyone including the merchant can verify the validity of the blind signature. The withdrawals are made by the bank by applying the blind signature of Schnorr [13] to a coin randomly selected by a customer and the payments are made by the customer by applying the group signature scheme of D. Yao and R. Tamassia [16] to the random coin.

\subsection{System Parameters}

This operation outputs the system parameters and public/private keys of users that will be used in the system.

- The group manager chooses a set of public parameters $Y=\left(G_{1}, G_{2}, e, P, H, H^{\prime}, H^{\prime \prime}\right)$, where $G_{1}$ and $G_{2}$ are groups of a large prime order $q, G_{1}$ is a gap group, $e: G_{1} \times G_{1} \rightarrow G_{2}$ is a bilinear map, $P$ is a generator of $G_{1}$ and $H:\{0,1\}^{*} \rightarrow G_{1}, H^{\prime}:\{0,1\}^{*} \rightarrow Z_{q}$ and $H^{\prime \prime}:\{0,1\}^{*} \times G_{1} \rightarrow Z_{q}$ are three collision-resistant hash functions. The group manager chooses his secret key $s_{A} \in Z_{q}^{*}$ and computes the public key $P_{A}=s_{A} P$.

- The customer chooses a secret $s_{u} \in Z_{q}^{*}$ as his private key and computes the product $P_{u}=s_{u} P$ as its public key.

- The bank selects a random secret $x_{b}$ from the interval $[1, q-1]$ and calculates the point $P_{b}=x_{b} P$. The public key of the bank is $P_{b}$ and the corresponding secret key is $x_{b}$.

The process for selecting the parameters and generating $G_{1}, G_{2}, q, e, P$ is given in [2].

\subsection{The Registration Protocol}

We assume that communication between the customer and the group manager is secure, i.e., private and authentic.

Any customer who wants to withdraw a coin from the bank has to interact with the group manager and obtains two type of certificates from the group manager. One is long-term group membership certificate, which certifies the customer's public key information. The other is one-time signing permit, which certifies the customer's one-time signing key information. The latter is used for issuing signatures in the payment protocol.

The registration protocol involves the customer and the group manager as follows:

1. A customer obtains a long-term group membership certificate Cert from the group manager. The group manager computes Cert $=s_{A} H\left(\right.$ info $\left.\| s_{u} P\right)$, where $s_{A}$ is the private key of the group manager, $s_{u} P$ is the customer's public key and info contains information such as group name and membership expiration date. Cert is given to the customer.

2. A customer also obtains one-time signing permits from the group manager. The customer randomly chooses a number of secrets $x_{1}, \ldots, x_{l}$ and computes one-time signing secret keys $x_{1} P, \ldots, x_{l} P$ and one-time signing public keys $s_{u} x_{1} P, \ldots, s_{u} x_{l} P$. The keys $s_{u} P$ and $s_{u} x_{i} P$ are sent to the group manager, for all $i=1, \ldots, l$. The customer also sends Cert to the group manager.

3. The group manager first checks if the customer with public key $s_{u} P$ is a valid group member. This is done by verifying the following equality:

$$
e(\operatorname{Cert}, P)=e\left(P_{A}, H\left(\text { info } \| s_{u} P\right)\right)
$$

where $P_{A}$ is the group manager's public key and $s_{u} P$ is the customer's public key. The protocol terminates if $e($ Cert,$P) \neq e\left(P_{A}, H\left(\right.\right.$ info $\left.\left.\| s_{u} P\right)\right)$. Then the group manager tests if $e\left(s_{u} x_{i} P, P\right)=e\left(s_{u} P, x_{i} P\right)$ for all $i=1, \ldots, l$. If the test fails, the protocol terminates. Otherwise, the group manager computes:

$$
S_{i}=s_{A} H\left(\text { infol|s } s_{u} P\right)
$$

for all $i=1, \ldots, l$. $S_{i}$ is an one-time signing permit and is given to the customer. The group manager adds the tuple $\left(s_{u} P, x_{i} P, s_{u} x_{i} P\right)$ to its record for all $i=1, \ldots, l$. 


\subsection{The Withdrawal Protocol}

We assume that communication between the customer and the bank is secure, i.e., private and authentic. The withdrawal protocol allows a customer to withdraw e-coins from the bank. After having open a bank account, the customer withdraws an e-coin from his account by using the blind signature. Therefore, the bank cannot link the e-coin to the identity of the customer but can debit to the account correctly. The withdrawal protocol involves the customer and the bank in which the customer withdraws an electronic coin from the bank. First, the customer proves his identity to the bank using the elliptic curve version of the signature scheme of Shao [14]. Then, the bank uses the elliptic curve version of the blind Schnorr signature scheme [13] to sign the e-coin.

The customer must perform the following protocol with the bank:

1. The customer sets his electronic cash requirement:

$$
m=H^{\prime}(\text { withdrawal require } \| I D)
$$

where $I D$ is the identity of the customer. Then, the customer chooses a random value $k_{u} \in[1, q-1]$ and signs the message $m$ using the elliptic curve version of the signature scheme of Shao [14]:

$$
\begin{aligned}
f & =H^{\prime}(m) \\
R & =k_{u} f P \\
h & =H^{\prime \prime}(m, R) \\
s & =k_{u}-h s_{u} .
\end{aligned}
$$

The customer sends $m$ and its signature $(h, s)$ to the bank.

2. The bank verifies the signature $(h, s)$ of the message $m$ :

(a) The bank first computes $f=H^{\prime}(m), R^{\prime}=f\left(h P_{u}+s P\right)$ and $h^{\prime}=H^{\prime \prime}\left(m, R^{\prime}\right)$.

(b) Then, the bank checks that the following equality holds:

$$
h=h^{\prime} .
$$

(c) If $h \neq h^{\prime}$ the protocol terminates.

3. Then, the bank uses the elliptic curve version of the blind Schnorr signature [13] to sign the e-coin: selects $k^{\prime} \in[1, q-1]$, computes the point $R^{\prime \prime}=k^{\prime} P$ and sends $R^{\prime \prime}$ to the customer.

4. The customer establishes a random coin $c$, randomly selects $\alpha, \beta \in[1, q-1]$, computes $R_{b}=R^{\prime \prime}+\alpha P+$ $\beta P_{b}, c_{b}=H^{\prime \prime}\left(c \| \alpha, R_{b}\right)$ and blinds the e-coin by computing $c^{\prime}=c_{b}-\beta \bmod q$. The customer sends the value $c^{\prime}$ to the bank.

5. The bank computes: $s^{\prime}=k^{\prime}-c^{\prime} x_{b} \bmod q$ and forwards $s^{\prime}$ to the customer.

6. The customer computes $s_{b}=s^{\prime}+\alpha \bmod q$. The pair $\left(c_{b}, s_{b}\right)$ is a valid e-coin signature issued by the bank.

7. The customer verifies the blind signature $\left(c_{b}, s_{b}\right)$ of the coin $c$, issued by the bank, by checking that the following equation holds:

$$
s_{b} P+c_{b} P_{b}=R_{b}
$$

8. The blind signature of the coin $c$ is the pair $\left(c_{b}, s_{b}\right)$.

The customer gets the coin $c$ from his account.

\subsection{The Payment Protocol}

The payment protocol involves the customer and the merchant and should be done through a secure channel (i.e., data privacy and integrity). In the proposed system, during payment the merchant does not communicate with the bank. After withdrawing e-coins, the customer can pay for what the merchant provided. Then the merchant verifies the validity of the received e-coins.

In order to sign the coin $c$, the customer uses the protocol of Yao and Tamassia [16]. The merchant first sends a challenge $c_{m}$ to the customer. Then, the customer produces a signature $S_{u}$ of the coin $c$ and merges the signature $S_{u}$ with his one-time signing permit $S_{i}$ associated with the secret $s_{u} x_{i}$. The details are as follows: 
1. The merchant sends challenge $c_{m}=H^{\prime}\left(I D_{m} \| T\right)$ to the customer, where $I D_{m}$ is the merchant's identity and $T$ is the recorded time of the transaction.

2. The customer computes:

$$
c_{u}=H^{\prime}\left(c\left\|c_{m}\right\| c_{b} \| s_{b}\right)
$$

3. The customer computes $S_{u}=s_{u} x_{i} H\left(c_{u}\right)$.

4. The customer computes the signature $S=S_{u}+S_{i}$, where $S_{i}=s_{A} H\left(\right.$ info $\left.\| s_{u} x_{i} P\right)$.

5. The customer sends $c, c_{u}$ and the signature $S=S_{u}+S_{i}$ of the coin $c$ to the merchant.

6. The merchant verifies the signature $S$ of the coin $c$ as follows:

(a) Computes the hash digest $H\left(c_{u}\right)$ and the hash digest $h^{\prime}=H\left(\right.$ info $\left.\| s_{u} x_{i} P\right)$ of one-time signing permit.

(b) The signature $S$ is accepted if

$$
e(S, P)=e\left(P_{A}, h^{\prime}\right) e\left(s_{u} x_{i} P, H\left(c_{u}\right)\right)
$$

If the test fails, the protocol terminates.

\subsection{The Deposit Protocol}

The deposit protocol permits the merchant to deposit the received e-coins to the bank. When receiving the deposited requirement from the merchant, the bank first verifies the validity of received e-coins and then credits the account of the merchant.

In on-line e-cash systems this protocol is part of the payment protocol as executed by the merchant. In our e-cash system, the deposit protocol is executed at a later moment, preferably in batch mode. The bank holds a record of spent cash to prevent double spending of e-cash. The bank cannot link deposited coins to a customer without collaboration from the group manager.

The deposit protocol involves the merchant and the bank as follows:

1. The merchant sends $c, c_{m}, c_{u}, c_{b}, s_{b}$ to the bank.

2. The bank verifies the signature as given in the equation (6).

3. After verification succeeds, the bank checks if $c$ obtained from the merchant exists in its database. If the coin $c$ is in the database of the bank, then the bank finds the signature $S^{\prime}$ for the deposited coin in its database and sends it to the merchant (detection of double spending).

4. If the merchant receives $S^{\prime}$ from the bank, he/she checks whether $S^{\prime}=S$. If $S^{\prime}=S$, then the merchant rejects performing protocol (double spending). Otherwise, the merchant sends $c_{u}$ and $T$ to the bank.

5. The bank verifies the validity of the signature $S$ using the equation (7).

6. If the signature $S$ of the coin $c$ is valid, then the bank accepts the coin $c$. Then, the bank will deposit the cash to the merchant's account and the merchant sends the goods to the customer. The bank stores $c$ and $\left(c_{u}, s_{u} x_{u} P\right)$ in its database.

7. If the bank finds out that $c$ and $\left(c_{u}, s_{u} x_{u} P\right)$ has been stored before but different $T$ and $c_{m}$, then the coin $c$ has been double spending. The bank performs the tracing protocol and detects the identity of the double spender with the help of the group manager.

\subsection{The Tracing Protocol}

The bank can legally trace the customer of a paid coin with the help of the group manager. The tracing protocol involves the bank and the group manager. Given a signature $S$ and its associated public information $P_{A}$ and $s_{u} x_{i} P$, the group manager verifies the signature $S$. If the signature $S$ is valid, the group manager can identify a customer's public key $s_{u} P$ from $s_{u} x_{i} P$ value, by consulting the customer group record. The details are as follows:

1. The bank sends $c_{u}$ and the signature $S$ of the coin $c$ to the group manager.

2. The group manager verifies the signature $S$ using the equation (7). 
3. The group manager can easily identify the customer from $s_{u} x_{i} P$. The group manager can provide a proof that it is indeed the customer's signature from the following equations:

$$
e\left(s_{u} x_{i} P, P\right)=e\left(s_{u} P, x_{i} P\right)
$$

4. The group manager searches through the group customer list to get the identity of the customer and sends it to the bank.

Similar to what is shown in the group signature scheme of Chen et al. [7], the group manager cannot misattribute a signature to frame the customer unless he can compute $b P$ given $q, P, a P$ and $d P$ which satisfies:

$$
a \equiv d b \bmod q
$$

The authors in [7] define this problem the Reversion of Computation Diffie-Hellman Problem. They prove that the Reversion of Computation Diffie-Hellman Problem is equivalent to Computational Diffie-Hellman Problem in $G_{1}$.

\section{Security and Efficiency Analysis}

In this section we discuss some aspects of security and efficiency of our off-line electronic payment system. We prove that our off-line electronic payment system is secure against tracing a honest customer by the bank and the proposed system is secure against forgery of the coin.

Theorem 1. Our off-line electronic payment system is secure against existential forgery of the coin c.

Proof: Long-term membership certificates, one-time signing permits and customer's signatures using one-time secret signing keys are generated by the sign protocol of the signature scheme of Boneh, Gentry, Lynn and Shacham [3]. The authors in [3] shown that their signature scheme is secure against existential forgery attacks. Therefore, if an adversary can forge any of these signatures, she can also forge signatures in the signature scheme of Boneh et al. [3]. Note that a signature computed with one-time secret signing key is in the form of $s_{u} x_{i} H\left(c_{u}\right)$, rather than $s_{u} H\left(c_{u}\right)$ as in the signature scheme [3]. It can be easily shown that if an adversary can forge a signature in a form of $s_{u} x_{i} H\left(c_{u}\right)$, then she can forge a signature in the form of $s_{u} H\left(c_{u}\right)$. Also, since the blind signature of Schnorr is secure against existential forgery, this allows only the legal bank to generate the signature for coin. As the hash function $H^{\prime}$ has the feature of collision free, the customer cannot find a value $c^{\prime} \neq c$ with $H^{\prime}\left(c^{\prime} \| c_{m}\right)=H^{\prime}\left(c \| c_{m}\right)$. Thus, our payment system satisfies unforgeability of the coin.

Theorem 2. The both valid signatures $S$ and $\left(c_{b}, s_{b}\right)$ in our payment system contain a proof of the group membership without revealing the identity of the customer.

Proof: A valid signature $S$ is obtained from an one-time signing permit of a customer and the customer's signature using the corresponding one-time signing key. That is $S=S_{i}+S_{u}$, where $S_{i}=s_{A} H\left(\right.$ info $\left.\| s_{u} x_{i} P\right)$ and $S_{u}=s_{u} x_{i} H\left(c_{u}\right)$. Because of the definition of signatures [3], a valid signature $S$ implies that $S_{i}$ is valid. This proves that the holder of key $s_{u} x_{i} P$ is a certified customer. A valid $S$ also means that $S_{u}$ is valid, therefore $S_{u}$ is generated with the secret key $s_{u} x_{i}$. Thus, $S$ contains a proof of the customer membership. Because the signing key $s_{u} x_{i}$ is one-time signing key and $x_{i}$ is chosen randomly by the customer, the identity of the customer is not revealed. Also, since $c_{u}=H^{\prime}\left(c\left\|c_{m}\right\| c_{b} \| s_{b}\right)$ and the blind signature $\left(c_{b}, s_{b}\right)$ of the coin $c$ can not give any information for the coin $c$, the bank can not link the blind coin with the identity of the customer.

Table 1: Storage space of the payment systems

\begin{tabular}{|l|l|l|l|l|l|}
\hline & Our system & Wang & Lee & Au & Canard \\
\hline \hline Withdrawal & 1120 bits & 1824 bits & 800 bits & 8160 bits & 6420 bits \\
\hline Payment & 480 bits & 1282 bits & 1304 bits & 5188 bits & 30740 bits \\
\hline Deposit & 960 bits & 3232 bits & 1656 bits & 5164 bits & 27648 bits \\
\hline
\end{tabular}


Table 2: Computation cost of the payment systems

\begin{tabular}{|l|l|l|l|l|l|}
\hline & Our system & Wang & Lee & $\mathrm{Au}$ & Canard \\
\hline \hline Withdrawal Protocol & & & & & \\
multi-EXP & 8 & 9 & 15 & 2156 & 5 \\
Pairing & 0 & 0 & 0 & 22 & 0 \\
\hline Payment Protocol & & & & & \\
multi-EXP & 2 & 11 & 9 & 34 & 1673 \\
Pairing & 3 & 0 & 0 & 14 & 0 \\
\hline Deposit Protocol & & & & & \\
multi-EXP & 0 & 5 & 7 & 10 & 14 \\
Pairing & 3 & 0 & 0 & 0 & 0 \\
\hline
\end{tabular}

Next, we evaluate the storage space and computational time of the costly operations. Table 1 and Table 2 summarize the storage space and computation cost respectively, of different protocols of our e-cash system and the schemes in [1], [4], [9] and [15]. The overall efficiency is improved in our electronic cash system compared to Au et al.'s system [1], Canard et al.'s system [4], Lee et al.'s system [9] and Wang et al.'s e-cash system [15] in terms of the storage space and the computation cost. Our system has a point $P$ of 160 bits and $q$ of 160 bits. The off-line e-cash system proposed by Lee et al. has a point $P$ of 160 bits and 160 bits prime $q$ and the system of Wang et al. has 160 bits prime $q$ and 321 bits prime $p$. Spending a coin in [15] requires 11 multi-based exponentiations and a total bandwidth of 1282 bits. The payment protocol in [9] requires 9 multi-based exponentiations and a total bandwidth of 1304 bits. For a moderate value $L=10$ and $t=40$, the payment protocol in [4] requires 1673 multibased exponentiations and a total bandwidth of 30740 bits. The payment protocol in [1] requires 34 multi-based exponentiations, 14 pairings and a total bandwidth of 5188 bits. In contrast, the payment protocol in our e-cash system requires 2 multi-based exponentiation, 3 pairings and a total bandwidth of 480 bits.

\section{Conclusions}

In this paper we presented a secure and efficient off-line electronic payment system based on bilinear pairing and group signature schemes. We used the group signature scheme of Yao and Tamassia and the blind signature of Schnorr. Because the amount of communication between customer and merchant is about 480 bits, the proposed off-line payment system can be used in the wireless networks with the limited bandwidth.

\section{Bibliography}

[1] M. Au, W. Susilo, Y. Mu, Practical anonymous divisible e-cash from bounded accumulators, Proceedings of Financial Cryptography and Data Security, Lecture Notes in Computer Science 5143 Springer-Verlag, pp. 287-301, 2008.

[2] D. Boneh and M. Franklin, Identity-based encryption from the Weil pairings. Advances in Cryptology-Crypto 2001, Lecture Notes in Computer Science 2139, Springer-Verlag, pp.213-229, 2001.

[3] D. Boneh, C. Gentry, B. Lynn, and H. Shacham, Aggregate and verifiably encrypted signatures from bilinear maps. In Advances in Cryptology - Eurocrypt'03, Lecture Notes in Computer Science 2656, Springer-Verlag, pp. 416-432, 2003.

[4] S. Canard, A. Gouget, Divisible e-cash systems can be truly anonymous, Proceedings of EUROCRYPT 2007, Lecture Notes in Computer Science 4515, Springer-Verlag, pp. 482-497, 2007.

[5] D. Chaum, Blind signature for untraceable payments. Proceddings of Eurocrypt'82, Plenum Press. pp.199203, 1983.

[6] D. Chaum, A. Fiat, M. Naor, Untraceable electronic cash, Proceedings of the Crypto'88, pp. 319-327, 1990.

[7] X. Chen,F. Zhang, K. Kim, A New ID-based Group Signature Scheme from Bilinear Pairings. Journal of Electronics, 23, pp. 892-900, 2006. 
[8] C. Fun, Ownership-attached unblinding of blind signatures for untraceable electronic cash, Information Science, 176(3), pp. 263-284, 2006.

[9] M. Lee, G. Ahn, J. Kim, J. Park, B. Lee, K. Kim, H. Lee, Design and implementation of an efficient fair off-line e-cash system based on elliptic curve discrete logarithm problem, Journal of Communications and Networks, 4(2), pp. 81-89, 2002.

[10] T. Okamoto, K. Ohta, Universal electronic cash, Proceedings of the 11th Annual International Cryptology Conference on Advances in Cryptology, pp. 324-337, 1992.

[11] T. Okamoto, An efficient divisible electronic cash scheme, Proceedings of Crypto'95, Lecture Notes in Computer Science 963, Springer-Verlag, pp. 438-451, 1995.

[12] C. Popescu, An Electronic Cash System Based on Group Blind Signatures. Informatica, 17(4), pp. 551-564, 2006.

[13] C.P. Schnorr, Efficient signature generation for smart cards, Journal of Cryptology, 4(1991), pp. 239-252, 1991.

[14] Zuhua Shao, A provably secure short signature scheme based on discrete logarithms, Information Sciences: an International Journal, vol.177(23), pp. 5432-5440, 2007.

[15] H. Wang, J. Cao, Y. Zhang, A flexible payment scheme and its role-based access control. IEEE Transactions Knowledge Data Engeneering, 17, pp. 425-436, 2005.

[16] D. Yao, R. Tamassia, Cascaded Authorization with Anonymous-Signer Aggregate Signatures. Proceedings of the Seventh Annual IEEE Systems, Man and Cybernetics Information Asssurance Workshop, USA, pp.84-91, 2006.

Horea Oros (b. August 22, 1977) received his PhD in Computer Science (2009) from "Babeş Bolyai” University of Cluj-Napoca, Romania. Since 2001 he is working within the Department of Mathematics and Computer Science, Faculty of Sciences, University of Oradea, Romania, where currently he is a lecturer. He also is lecturer at Agora University of Oradea. He co-authored three books in the filed of computer science and published 19 articles in several journals and proceedings of prestigious international conferences. His main research interest is in the field of cryptology and computer security.

Constantin Popescu (b. October 21, 1967) received his PhD in Computer Science (2001) from "Babeş Bolyai" University of Cluj-Napoca, Romania. Since 2005 he is a professor at the Department of Mathematics and Computer Science, University of Oradea, Romania. His research interests include cryptography, network security, group signatures, security protocols and electronic payment systems. He co-authored 7 books in the field of computer science and published 49 articles in several journals and proceedings of prestigious international conferences. He is reviewer for 10 journals and several prestigious international conferences. 